\title{
Causes of hOCT1-Dependent Cholangiocarcinoma Resistance to Sorafenib and Sensitization by Tumor-Selective Gene Therapy
}

\author{
Elisa Lozano, ${ }^{1,7}$ Rocio I.R. Macias (DD,${ }^{1,7}$ Maria J. Monte, ${ }^{1,7}$ Maitane Asensio, ${ }^{1}$ Sofia del Carmen, ${ }^{2}$ Laura Sanchez-Vicente, ${ }^{1}$ \\ Marta Alonso-Peña, ${ }^{1}$ Ruba Al-Abdulla, ${ }^{1}$ Patricia Munoz-Garrido, ${ }^{3}$ Letizia Satriano, ${ }^{3}$ Colm J. O’Rourke, ${ }^{3}$ Jesus M. Banales, ${ }^{4,7}$ \\ Matias A. Avila, ${ }^{5,7}$ Maria L. Martinez-Chantar, ${ }^{6,7}$ Jesper B. Andersen (D) ${ }^{3}$ Oscar Briz, ${ }^{1,7}$ and Jose J.G. Marin (iD) 1,7
}

\begin{abstract}
Although the multi-tyrosine kinase inhibitor sorafenib is useful in the treatment of several cancers, cholangiocarcinoma (CCA) is refractory to this drug. Among other mechanisms of chemoresistance, impaired uptake through human organic cation transporter type 1 (hOCT1) (gene SLC22A1) has been suggested. Here we have investigated the events accounting for this phenotypic characteristic and have evaluated the interest of selective gene therapy strategies to overcome this limitation. Gene expression and DNA methylation of SLC22A1 were analyzed using intrahepatic (iCCA) and extrahepatic (eCCA) biopsies (Copenhagen and Salamanca cohorts; $n=132$ ) and The Cancer Genome Atlas (TCGA)-CHOL ( $\mathrm{n}=36$ ). Decreased bOCT1 mRNA correlated with hypermethylation status of the SLC22A1 promoter. Treatment of CCA cells with decitabine (demethylating agent) or butyrate (histone deacetylase inhibitor) restored hOCT1 expression and increased sorafenib uptake. MicroRNAs able to induce bOCT1 mRNA decay were analyzed in paired samples of TCGA-CHOL $(n=9)$ and Copenhagen $(n=57)$ cohorts. Consistent upregulation in tumor tissue was found for miR-141 and miR-330. High proportion of aberrant hOCT1 mRNA splicing in CCA was also seen. Lentiviral-mediated transduction of eCCA (EGI-1 and TFK-1) and iCCA (HuCCT1) cells with hOCT1 enhanced sorafenib uptake and cytotoxic effects. In chemically induced CCA in rats, reduced rOct1 expression was accompanied by impaired sorafenib uptake. In xenograft models of eCCA cells implanted in mouse liver, poor response to sorafenib was observed. However, tumor growth was markedly reduced by cotreatment with sorafenib and adenoviral vectors encoding hOCT1 under the control of the BIRC5 promoter, a gene highly up-regulated in CCA. Conclusion: The reason for impaired hOCT1-mediated sorafenib uptake by CCA is multifactorial. Gene therapy capable of selectively inducing hOCT1 in tumor cells can be considered a potentially useful chemosensitization strategy to improve the response of CCA to sorafenib. (Hepatology 2019;70:1246-1261).
\end{abstract}

iliary tract cancers are a type of heterogeneous tumors that include cholangiocarcinoma (CCA), both intrahepatic (iCCA) and extrahepatic (eCCA), and gallbladder cancer, with diverse phenotypic characteristics. ${ }^{(1)}$ CCA, whose incidence is increasing worldwide, is currently the

Abbreviations: CCA, cholangiocarcinoma; DAC, decitabine (5-aza-2'-deoxycytidine); eCCA, extrahepatic CCA; DHE, dibydroethidium; DMSO, Dimethyl sulfoxide; EGFP, enhanced green fluorescent protein; HCC, hepatocellular carcinoma; hOCT1, human organic cation transporter type 1; HDAC, histone deacetylases; $i C C A$, intrahepatic CCA; miR-seq, miRNA-sequencing; $M O C$, mechanism of chemoresistance; NT, nontumor; OCT1, organic cation transporter 1; RBPs, RNA binding proteins; SNP, single nucleotide polymorphism; STAT3, signal transducer and activator of transcription 3; T, tumor; TAA, thioacetamide; TCGA, The Cancer Genome Atlas; TEA, tetraethylammonium; TKI, tyrosine kinase inhibitor; TSA, trichostatin A; UTR, untranslated region.

Received June 27, 2018; accepted April 4, 2019.

Additional Supporting Information may be found at onlinelibrary.wiley.com/doi/10.1002/hep.30656/suppinfo.

Supported by the Carlos III Institute of Health, Spain (Grants PI15/00179, PI16/00598 and PI18/01075 cofinanced by European Regional Development Fund); Ministry of Science and Innovation, Spain (SAF2013-40620-R and SAF2016-75197-R); "Asociación Española Contra el Cancer," Spain (AECC-2017); "Junta de Castilla y León," Spain (SA015U13 and SA063P17); "Fundación Samuel Solórzano Barruso," Spain (FS/10-2014, FS/08-2017 and FS/13-2017); "Fundación Mutua Madrileña," Spain (Call 2015); "Fundación Vasca de Innovación e Investigación Sanitarias," Spain (Bioef BIO15/CA/011); "Ministerio de Economía, Industria y Competitividad," Spain (SAF2017-87301-R); "Gobierno Vasco-Departamento de Salud," Spain (2013111114); and Basque Foundation for Innovation and Health Research: EiTB Maratoia, 
second most frequent primary hepatic malignancy after hepatocellular carcinoma (HCC). Surgical resection constitutes the best option for complete cure. However, owing to the lack of accurate noninvasive CCA markers and to the fact that these tumors grow up asymptomatically, they are often detected at an unresectable advanced stage. ${ }^{(2,3)}$ This partly accounts for the very poor prognosis of this cancer, from which most patients die within 12 months after diagnosis. Classical chemotherapy offers a 5-year survival rate lower than $10 \%$, which is due to the negligible degree of response of all types of CCA to available chemotherapeutic regimens. ${ }^{(4,5)}$ Gemcitabine plus cisplatin has become the reference regimen for systemic chemotherapy in patients with biliary tract cancers ${ }^{(6)}$; nonetheless, this chemotherapy is poorly effective. Therefore, it is essential to understand the molecular bases of the strong chemoresistance of CCA and to seek new therapeutic approaches. Despite their heterogeneity regarding several clinical and biological aspects, biliary cancers share their marked chemoresistance. Among the so-called targeted therapies, sorafenib, a multitargeted tyrosine kinase inhibitor (TKI) that blocks the activity of Raf serine/threonine kinase isoforms, as well as vascular endothelial growth factor receptor-2/3, platelet-derived growth factor receptor, c-KIT, FLT-3, and RET, to inhibit tumor angiogenesis and tumor cell proliferation, ${ }^{(7,8)}$ has been approved for the treatment of $\mathrm{HCC}{ }^{(9)}$ Sorafenib is one of the few TKIs that is active even against mutated BRAF, which appears with a high frequency in CCA, especially in iCCA. Moreover, sorafenib has been reported to have some anticancer activity against CCA in experimental models both in vitro and in vivo, which has not been consistently confirmed in clinical studies. ${ }^{(10-14)}$ The lack of response of patients with CCA to sorafenib may be the result of the combined action of several mechanisms of chemoresistance (MOCs). ${ }^{(5)}$ We have demonstrated that the organic cation transporter 1 (hOCT1, SLC22A1 gene) can play a key role in sorafenib effectiveness because the mechanism of action of this drug depends on its access to the intracellular domains of the tyrosine kinases that are inhibited by sorafenib. ${ }^{(15)}$ Moreover, hOCT1 mediates the uptake of this drug by target cells. ${ }^{(15)}$ Of note, it has been demonstrated that decreased expression of hOCT1 constitutes a shared characteristic of liver tumors (HCC, CCA, and hepatoblastoma). ${ }^{(16)}$ In addition, not all synthesized bOCT1 mRNA is translated into functional protein because of the high proportion of inactive variants that are generated as a result of alternative splicing mechanisms or single-nucleotide polymorphisms (SNPs). ${ }^{(15)}$

Spain (BIO15/CA/O16/BD). E.L. was supported by a postdoctoral contract by "University of Salamanca," Spain (2018). M.A. and M.A.P. were supported by a predoctoral contract by "Ministry of Education, Culture and Sports," Spain (BOE-A-2015-9456). R.A.A. was supported by a predoctoral contract funded by the "Junta de Castilla y León, Fondo Social Europeo" (EDU/828/2014). J.B.A. is supported by the Danish Cancer Society (R98-A6446); Novo Nordisk Foundation (14040); P.M.G.: European Association for the Study of the Liver (EASL) individual Sheila Sherlock Postdoc Fellowship, Marie Sklodowska-Curie Fellowships (MirChol and Epi-Target). C.J.O. is funded by MSCA postdoc fellowship.

(C) 2019 by the American Association for the Study of Liver Diseases.

View this article online at wileyonlinelibrary.com.

DOI 10.1002/hep.30656

Potential conflict of interest: Nothing to report.

\section{ARTICLE INFORMATION:}

From the ${ }^{1}$ Experimental Hepatology and Drug Targeting (HEVEFARM), IBSAL, University of Salamanca, Salamanca, Spain; ${ }^{2}$ Salamanca University Hospital, IBSAL, University of Salamanca, Salamanca, Spain; ${ }^{3}$ Biotech Research and Innovation Centre, Department of Health and Medical Sciences, University of Copenhagen, Copenhagen, Denmark; ${ }^{4}$ Department of Hepatology and Gastroenterology, Biodonostia Health Research Institute, Donostia University Hospital, University of the Basque Country (UPV/EHU), Ikerbasque, San Sebastian, Spain; ${ }^{5}$ Hepatology Programme, Center for Applied Medical Research (CIMA), IDISNA, University of Navarra, Pamplona, Spain; ${ }^{6}$ Liver Disease Laboratory, CIC bioGUNE, Technology Park of Vizcaya, Vizcaya, Spain; ${ }^{7}$ National Institute for the Study of Liver and Gastrointestinal Diseases (CIBERehd), Carlos III National Health Institute, Madrid, Spain.

\section{ADDRESS CORRESPONDENCE AND REPRINT REQUESTS TO:}

Jose J.G. Marin, Ph.D.

Department of Physiology and Pharmacology

Campus Miguel de Unamuno E.I.D. S-09
37007 Salamanca, Spain

E-mail: jjgmarin@usal.es

Tel.: +1-34-663182872 
In the present study, we have evaluated the hypothesis that mechanisms that cause impaired hOCT1 expression/function in CCA may be involved in the lack of response of these tumors to sorafenib, and we have investigated the usefulness of epigenetic manipulation and gene therapy in order to sensitize CCA to this drug by selectively enhancing hOCT1 expression in cancer cells under the control of a tumor-specific promoter.

\section{Materials and Methods}

\section{HUMAN SAMPLES AND DATA}

Whole transcriptome profiling was performed using human Ref-8v2 BeadChips (Illumina) on $68 \mathrm{iCCA}$ and 36 eCCA surgical specimens $(\mathrm{T})$ and 60 samples from adjacent nontumor tissue (NT), as described (GEO: GSE26566; "Copenhagen cohort" of patients). ${ }^{(17)}$ For a subset of these patients ( $\mathrm{T}=48, \mathrm{NT}=41)$, DNA methylation profiling was performed using Infinium HumanMethylation27 BeadChip(Illumina). The methylation status of the hOCT1 promoter was measured using beta $(\beta)$-value metric (range: $0-1 ; 0 \%-100 \%$ methylation) (see detailed description in Supporting Information). To further investigate the degree of DNA hypermethylation in hOCT1, level 1 Infinium HumanMethylation450 BeadChip (Illumina Inc.) data $(\mathrm{T}=36$ and $\mathrm{NT}=9)$ obtained from The Cancer Genome Atlas (TCGA)-CHOL consortium ${ }^{(18)}$ were analyzed. For an extended subset of the "Copenhagen cohort," miRNA-sequencing (miR-seq) data were also available for 57 paired T and NT samples and 22 normal livers. For some analyses, we have used specimens of iCCA $(n=16)$ and eCCA $(n=12)$ and paired NT samples $(n=17)$ obtained after tumor resection at University Hospital of Salamanca ("Salamanca cohort," see Supporting Table S1). Research protocols were approved by the Ethical Committees for Clinical Research of supporting institutions, and all patients signed written consents for the use of their samples for biomedical research.

\section{ANIMALS AND IN VIVO EXPERIMENTS}

Male Wistar rats (University of Salamanca Animal House) and female nude mice (Swiss nu/nu; Charles
River Laboratories, Barcelona) were used. Nude mice were maintained under pathogen-free environment and handled under stringent sterile conditions. The animals were fed on standard rat or mouse chow (Panlab, Madrid) and water ad libitum. Temperature $\left(20^{\circ} \mathrm{C}\right)$ and the light/dark cycle (12 hours: 12 hours) were controlled and the protocols were approved by the Ethical Committee of the University of Salamanca. To study sorafenib uptake by CCA, this tumor was induced in rats by including $0.05 \%$ thioacetamide (TAA) in the drinking water for 30 weeks $^{(19)}$ (Supporting Information). Xenografts of CCA in nude mice were generated by subcutaneous injection of $\approx 1 \times 10^{6}$ human EGI- 1 eCCA cells to donor nude mice under isoflurane anesthesia. EGI-1 cells were selected because of their acceptable tumorigenesis ability in vivo as compared with TFK-1 and HuCCT1 cells. Seven weeks later, generated CCA tumors were resected and dissected into $\sim 1$ $\mathrm{mm}^{3}$ pieces that were implanted under anesthesia in the livers of several host nude mice. ${ }^{(20)}$ The following day, the animals were randomly divided into four groups for the coadministration of control adenoviruses (Ad-Mock: Ad-BIRC5pr-enhanced green fluorescent protein [EGFP]) or adenoviruses containing Ad-BIRC5pr-hOCT1-EGFP plus sorafenib or the vehicle alone (saline). Additional control mice were used to measure serum levels of routine biochemical parameters for comparative purposes. Adenoviral particles (VP) were injected every 5 days $\left(4 \times 10^{7} \mathrm{VP} /\right.$ mice in each administration, intravenously through the tail vein). Sorafenib (10 mg/kg body weight) or saline were administered (intraperitoneally) twice per week. At the end of the experiment (after 2 months), the animals were anesthetized with sodium pentobarbital to measure the tumor volume and to collect tissues and blood samples. Serum levels of routine biochemical parameters were determined in a dry chemistry automated analyzer Spotchem EZ SP-4430 (Arkray Factory, A. Menarini Diagnostics, Badalona, Spain).

\section{LENTIVIRAL AND ADENOVIRAL VECTORS}

The human OCT1 open reading frame (ORF) was amplified from total RNA isolated from healthy liver by reverse transcription followed by high-fidelity PCR using AccuPrime Pfx DNA polymerase (Life Technologies) and specific primers (Supporting Table S2). OCT1 ORF 
was cloned into the PacI site of the pWPI lentiviral vector (that contains the constitutive $\mathrm{EF} 1 \alpha$ promoter). Recombinant lentiviruses production and lentiviral transduction are described in the Supporting Information.

The promoter region of the BIRC5 gene (BIRC5pr, 1467-bp zone of the 5'-flanking region) was cloned from human hepatoma PLC/PRF/5 (Alexander) cells using AccuPrime Pfx DNA polymerase, and specific oligonucleotide primers (Supporting Table S2). The cloning procedure is described in detail in the Supporting Information.

\section{IN VITRO EXPERIMENTS}

Cells transduced with lentiviral vectors (LentMOCK or Lent-OCT1) were seeded onto 96-well plates at subconfluence (5,000 to 7,500 cells/dish). After 24 hours, the cells were exposed to $5 \mu \mathrm{M}$ sorafenib for 6 hours. The formazan test using thiazolyl blue tetrazolium bromide (Sigma-Aldrich) was used to determine cell viability 66 hours later.

The transport function of hOCT1 was measured by the uptake of organic cations, such as dihydroethidium (DHE, $5 \mu \mathrm{M}$ ) by flow cytometry and $\left[{ }^{14} \mathrm{C}\right]$-tetraethylammonium (TEA; $150 \mu \mathrm{M}$ ) by radioactivity determination. Specific hOCT1 inhibition was determined using quinine $(250 \mu \mathrm{M})$. Sorafenib $(5 \mu \mathrm{M})$ uptake was also determined using an adaptation of a published method using high-performance liquid chromatography-tandem mass spectrometry (HPLC-MS/MS). ${ }^{(15)}$ In uptake experiments, the results were corrected by protein content. ${ }^{(21)}$

The role of miRNAs potentially involved in regulating hOCT1 expression was studied in EGI-1 and TFK-1 cells, using lentivirus designed to mimic the pre-miRNA (hsa-mir-141/330/1468) sequence including the loop (Supporting Information).

\section{QUANTITATIVE RT-PCR (RT-QPCR)}

Total RNA extraction from cells and tissues and retro-transcription were performed as described ${ }^{(16)}$ (Supporting Information). The primer oligonucleotide sequences to carry out QPCR are described in Supporting Table S3. The results of mRNA abundance of target genes in each sample were normalized on the basis of glyceraldehyde 3-phosphate dehydrogenase $(G A P D H)$, hypoxanthine-guanine phos- phoribosyltransferase (HPRT1), or rat $\beta$-actin (ACTB) mRNA abundance.

\section{IMMUNOFLUORESCENCE AND IMMUNOBLOTTING ASSAYS}

Immunofluorescent staining was performed on tissue cryosections air-dried and fixed in cold methanol using appropriate antibodies against hOCT1, platelet/ endothelial cell adhesion molecule 1 , and $\mathrm{Na}^{+} / \mathrm{K}^{+}$ATPase. Nuclei were counterstained with 4',6-diamidino-2-phenylindole (DAPI). Confocal laser-scanning microscopy was performed using a Leica TCS SP2 confocal microscope. Immunoblotting analyses of cell lysates were carried out in 10-12\% sodium dodecyl sulfate-polyacrylamide gel electrophoresis, loading $30 \mu \mathrm{g}$ of protein per lane. Appropriate primary antibodies for survivin and GAPDH were diluted in phosphate-buffered saline-Tween. Immunoreactive protein bands were visualized by enhanced chemiluminescence (Amersham Pharmacia Biotech) after incubation with appropriate secondary antibodies (immunoglobulin G-horseradish peroxidase linked). See Supporting Information for antibodies characteristics and conditions.

\section{DETERMINATION OF ALTERNATIVE SPLICING}

Based on previous reports of alternative spliced hOCT1 variants, ${ }^{(15)}$ we designed primers annealing in exon 6 (Forward) and exon 11 (Reverse) that are shared by all hOCT1 isoforms (Supporting Table S3). PCR was carried out with Platinum-Taq DNA polymerase (Life Technologies) using 30 cycles of amplification. The presence and size of the PCR products were determined by gel electrophoresis, and a semiquantitative determination of the amount of each splicing variant was carried out by densitometry of the bands using a LAS-4000 luminescent image analyzer.

\section{STATISTICAL ANALYSES}

Results were statistically analyzed using GraphPad program. For comparisons between two groups, parametric paired $t$ test or Student $t$ test and nonparametric Mann-Whitney U test were used. After analysis of variance, Bonferroni method of multiple-range testing was used to calculate the statistical significance of differences among groups. 


\section{Results}

\section{ROLE OF DNA METHYLATION IN HOCT1 DOWN-REGULATION}

The relationship between hOCT1 expression and the methylation status of the $S L C 22 A 1$ promoter was analyzed in resected CCA specimens from two different cohorts of patients: (1) TCGA-CHOL, including TCGA Infinium 450k data, and (2) the "Copenhagen cohort." We have confirmed hOCT1 down-regulation in CCA versus peritumor tissue in both cohorts (Fig. 1A,C). hOCT1 promoter was significantly hypermethylated in CCA (using the probe cg27292431, corresponding to exon 1 of SLC22A1) compared with peritumor tissue (Fig. 1B,D). Owing

TCGA Cohort
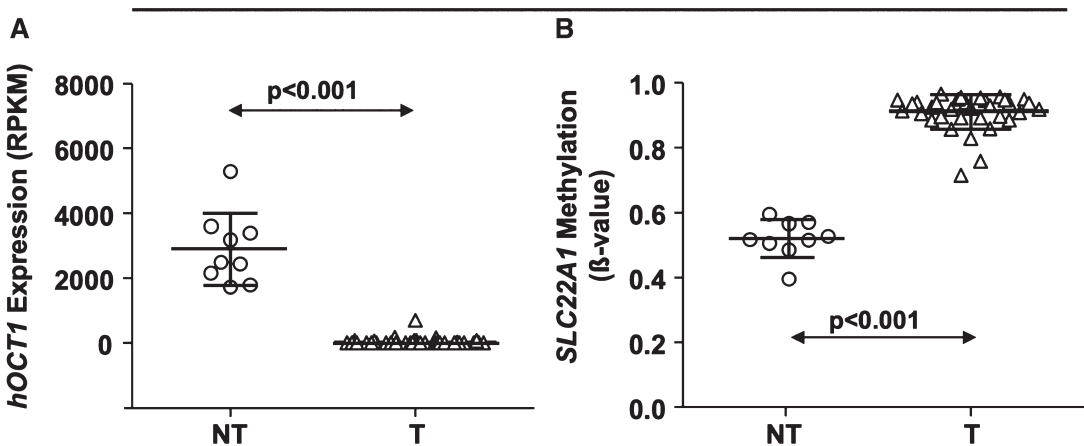

Copenhagen Cohort
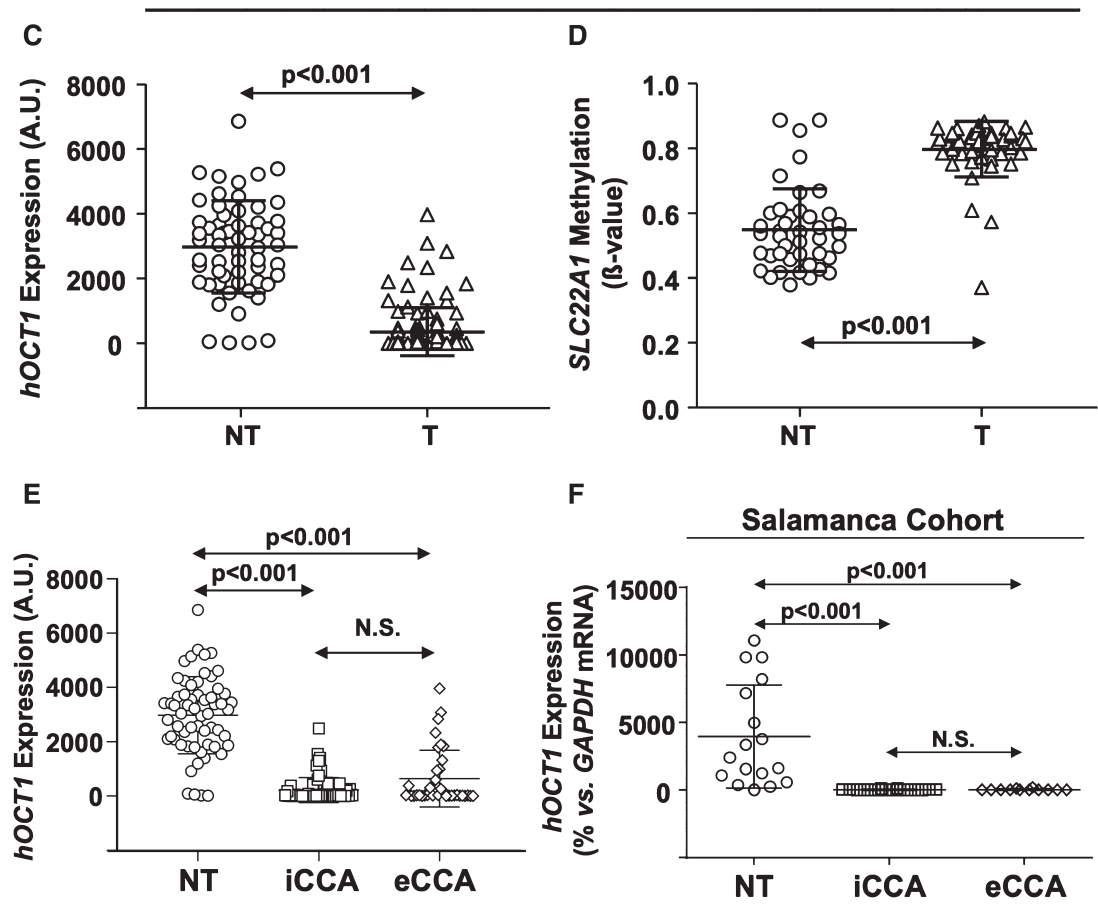

FIG. 1. hOCT1 expression and promoter methylation in CCA. Levels of hOCT1 mRNA (A,C) determined by microarray and methylation status $(\mathrm{B}, \mathrm{D})$ in tumor $(\mathrm{T})$ compared with paired non-tumor $(\mathrm{NT})$ tissue analyzed in $(\mathrm{A}, \mathrm{B}) \mathrm{TCGA}-\mathrm{CHOL}(\mathrm{NT}, \mathrm{n}=9$; $\mathrm{T}, \mathrm{n}=36)$ and $(\mathrm{C}, \mathrm{D})$ Copenhagen cohort $(\mathrm{NT}, \mathrm{n}=60 ; \mathrm{T}, \mathrm{n}=104)$. Methylation was determined using cg27292431 probe at exon 1 of SLC22A1. Values are individual measurements of T (open triangles) and NT (open circles) samples or mean \pm SD (solid lines). Comparison between T and NT was carried out using Mann-Whitney U test. Separate analysis of hOCT1 expression in iCCA and eCCA tumors and adjacent NT liver tissue in (E) the Copenhagen cohort (iCCA, $n=68$; eCCA, $n=36 ; \mathrm{NT}, \mathrm{n}=60)$ and $(\mathrm{F})$ the Salamanca cohort (iCCA, $\mathrm{n}=16$; eCCA, $\mathrm{n}=12 ; \mathrm{NT}, \mathrm{n}=17$ ). Values are represented as mean \pm SD. Abbreviations: A.U., arbitrary units; RPKM, reads per kilobase mapped. N.S., no significant difference $(P>0.05)$. 
to the importance of the location of $\mathrm{CpG}$ dinucleotide hypermethylation in relationship to gene expression, we analyzed $450 \mathrm{k}$ data from TCGA-CHOL, which provides increased hOCT1 promoter coverage. Accordingly, we uncovered three additional hypermethylated probes mapping to the transcriptional start site and 5'-untranslated region (UTR) of hOCT1 (Supporting Fig. S1). When, both in the "Copenhagen cohort" (Fig. 1E) and the "Salamanca cohort" (Fig. 1F), hOCT1 expression was analyzed separately in iCCA and eCCA, similar marked down-regulation in both types of tumors was found.

\section{IN VITRO hOCT1 EXPRESSION}

To investigate the functional impact of SLC22A1 hypermethylation, CCA cell lines also with markedly reduced bOCT1 mRNA levels ${ }^{(15,16)}$ were treated with decitabine (5-aza-2'-deoxycytidine) (DAC). This DNA demethylating agent restored hOCT1 expression in EGI-1 (Fig. 2A) and TFK-1 (Fig. 2B) cells. Because acetylation of histones facilitates gene expression and histone deacetylases (HDACs) are overexpressed in CCA cells, which leads to a reduced expression of genes involved in differentiation, ${ }^{(2)}$ we evaluated whether HDAC inhibitors (HDACI) could affect bOCT1 mRNA expression in CCA cells. Sodium butyrate induced hOCT1 up-regulation in both EGI-1 (Fig. 2C) and TFK-1 (Fig. 2D) cells. The magnitude of the effect after 5 days of treatment was DAC>butyrate in EGI-1 cells but butyrate>DAC in TFK-1 cells. Other HDACIs, such as phenyl butyrate, increased bOCT1 mRNA levels, but only in TFK-1 cells and to a lesser extent than butyrate, whereas trichostatin A had no significant effect in any of these cell lines (Fig. 2C,D). Using EGI-1, in which DAC effect was stronger, it was demonstrated that the recovery of hOCT1 expression was accompanied by enhanced sorafenib uptake (Fig. 2E).

\section{ROLE OF microRNA IN hOCT1 DOWN-REGULATION}

In a separate study, we have carried out in silico analysis of microRNA-induced $b O C T 1$ mRNA decay. Among six selected microRNAs, only three showed activity in HepG2 hepatoma cells. ${ }^{(23)}$ Here, we have shown that the same microRNAs, i.e., 141, 1468, and 330, reduced bOCT1 mRNA in EGI-1 (Fig. 2F) and TFK-1 (Fig. 2G) cells. The expression of these three
microRNAs in paired $\mathrm{T}$ and NT specimens from both TCGA-CHOL and the extended "Copenhagen cohort" was analyzed and compared with that of bOCT1 (Fig. 3). Both miR-141 and miR-330 were consistently up-regulated in both groups of CCA. In the same paired specimens, bOCT1 mRNA was consistently decreased (Fig. 3F,L). In contrast, there was a discrepancy between both series regarding changes in miR-1468 expression (Fig. 3B,H).

\section{ABERRANT SPLICING}

To quantify the importance of aberrant splicing in overall hOCT1 expression/function, we evaluated the presence of splicing variants by PCR using specific primers to amplify the hOCT1 amplicon between the exons 6 and 11 (Fig. 4A). The length of the amplified fragment was used to distinguish wild-type from shorter variants (Fig. 4B,C). Measurement by densitometry of the abundance of splicing variants revealed a higher proportion of aberrant forms in CCA (both in biopsies and in cell lines) than in healthy liver. Interestingly, aberrant splicing was also present in peritumor tissue (Fig. 4D).

\section{ROLE OF mRNA STABILITY/DECAY PROTEINS}

To investigate whether changes in the balance of RNA binding proteins (RBPs) involved in mRNA stability/decay could affect the levels of bOCT1 mRNA in CCA, the expression of genes involved in mRNA decay ( $A U F 1, B R F 1, B R F 2, C U G B P, F B P 2$, and $T T P)$ or mRNA stability $(H u R)$ was measured in paired samples of tumor and peritumor tissue ("Salamanca cohort"). We found no significant difference between T and NT tissue for any of these genes (Supporting Fig. S2A-F), except for that encoding tristetraprolin (TTP) (Supporting Fig. S2G), that was down-regulated in CCA samples. Similar results were found when the expression levels of RBP genes were determined in CCA cell lines (data not shown).

\section{hOCT1 DOWN-REGULATION CORRELATES WITH DECREASED SORAFENIB UPTAKE BY CCA TUMORS}

A rat model of chemically induced $\mathrm{CCA}^{(19)}$ was used to study whether down-regulation of this 
A

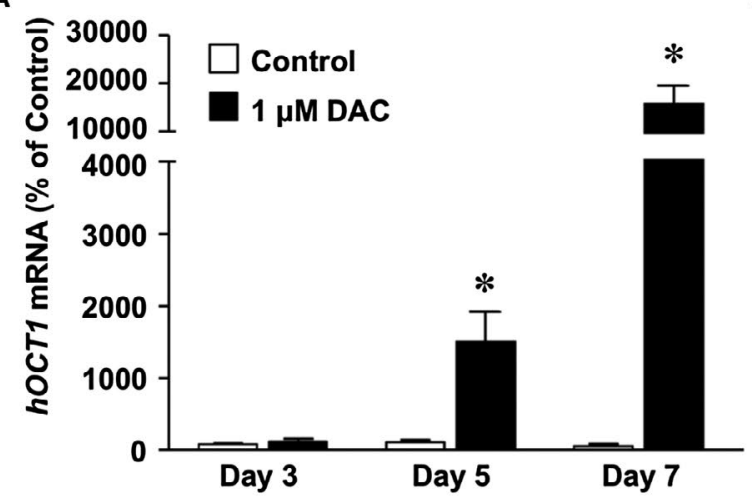

C

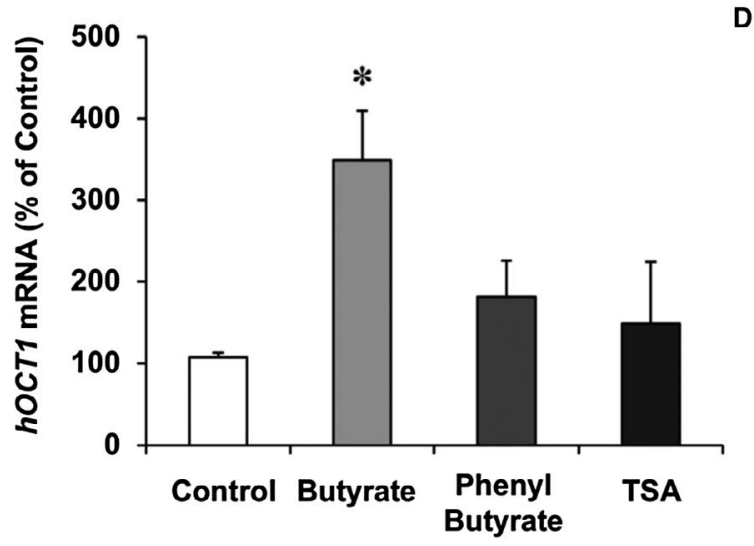

E

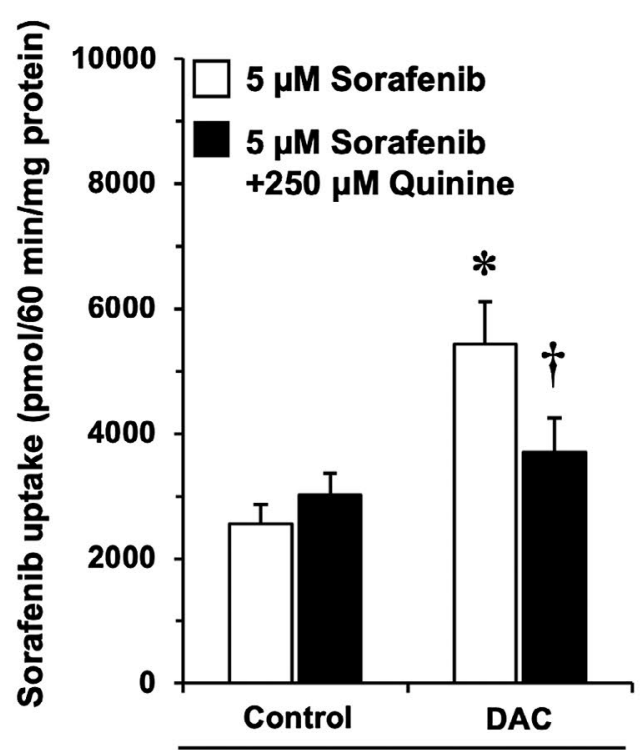

EGI-1 Cells
B
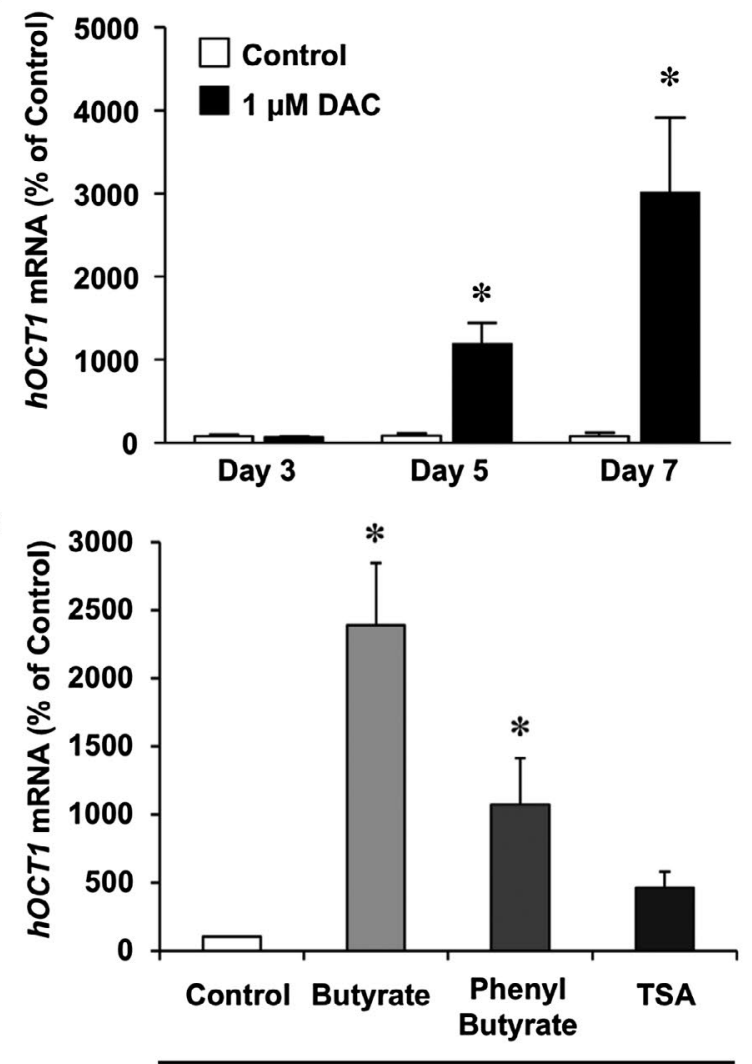

G TFK-1 Cells

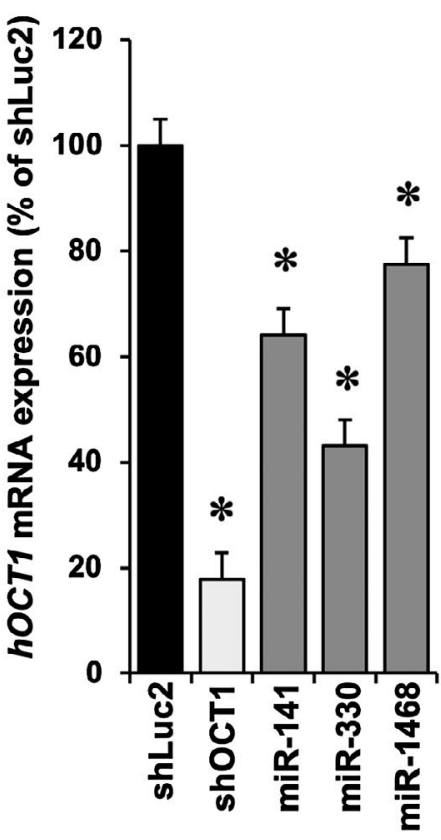

EGI-1 Cells

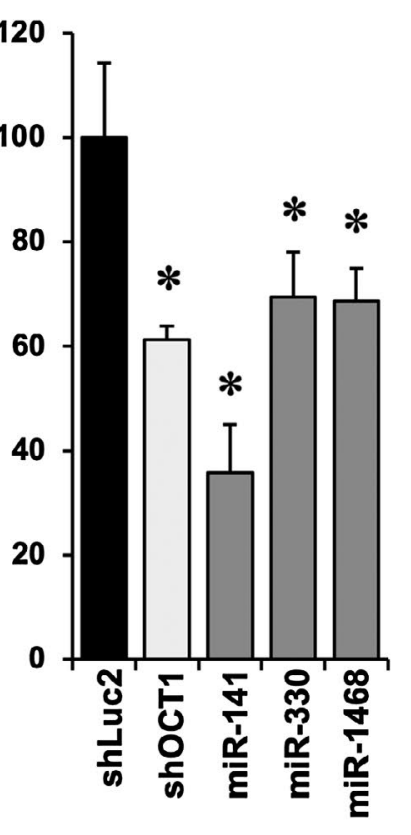

TFK-1 Cells 
FIG. 2. Effect of hypomethylating agents and histone deacetylase inhibitors on hOCT1 expression/function. hOCT1 mRNA levels in (A) EGI-1 and (B) TFK-1 CCA cells were measured after exposure to $1 \mu \mathrm{M}$ of DAC for 3, 5, or 7 days. In Control groups, a similar amount of the vehicle (dimethyl sulfoside, DMSO) alone was added to the culture medium. hOCT1 mRNA levels in (C) EGI-1 and (D) TFK-1 CCA cells after incubation with histone deacetylase inhibitors: $5 \mathrm{mM}$ sodium butyrate, $5 \mathrm{mM}$ phenyl butyrate, or $150 \mathrm{nM}$ trichostatin A (TSA). (E) To evaluate the effect of DAC treatment (1 $\mu \mathrm{M}$ for 5 days) on the ability of EGI-1 cells to take up sorafenib, EGI-1 cells were incubated with $5 \mu \mathrm{M}$ sorafenib with or without $250 \mu \mathrm{M}$ quinine for 1 hour, and its levels in the cells were measured by HPLC-MS/MS. Evaluation of the ability of three miRNAs to induce bOCT1 mRNA decay in (F) EGI-1 and (G) TFK-1 cells. CCA cells were transduced with lentiviral vectors able to induce the expression of short-hairpin RNA against luciferase (sh-Luc2, Control), or against hOCT1 (sh-hOCT1), or one of the three microRNA selected from their predicted interaction with pre-mRNA by in silico analysis, and 1 day after transduction, cells were treated with $1 \mu \mathrm{M}$ DAC for 5 days to maximize hOCT1 expression. Values are mean $\pm \mathrm{SD}(\mathrm{n}=4) .{ }^{*}, P<0.05$ on comparing with control. $\dagger, P<0.05$ on comparing with results obtained in absence of quinine.

transporter results in impaired uptake of sorafenib by CCA tumors in vivo (Fig. 5). Sorafenib was intravenously administered to CCA-bearing rats. Samples from both $\mathrm{T}$ and NT tissue were collected 1 hour later, and their sorafenib content was determined using HPLC-MS/MS. The results indicated that peritumor tissue, which maintained higher Oct1 levels in comparison with CCA (Fig. 5D), was able to efficiently take up sorafenib (Fig. 5E). In contrast, in the tumors, the reduced expression of Oct1 (Fig. 5D) was accompanied with a consistent and significant decrease in sorafenib content (Fig. 5E).

\section{RELATIONSHIP BETWEEN hOCT1 EXPRESSION AND SORAFENIB UPTAKE/ACTIVITY}

To evaluate whether the experimental overexpression of hOCT1 could be used to increase the cytotoxic effect of sorafenib, eCCA (TFK-1 and EGI-1) and iCCA (HuCCT1) cells were transduced using lentivirus, either empty (MOCK) or containing hOCT1 coding sequence (Lent-OCT1). The high efficacy of transduction, as analyzed by counting EGFP-positive cells $(\approx 95 \%, \approx 60 \%$, and $\approx 80 \%$ in TFK-1, EGI-1, and HuCCT1 cells, respectively), resulted in a marked increase in bOCT1 mRNA expression (Fig. 6A-C). Consequently, hOCT1transduced cells showed enhanced ability to take up, in a quinine-sensitive manner, organic cations, such as DHE (Fig. 6D-F) and TEA (Supporting Fig. S3). Moreover, in comparison with MOCK-transduced cells, hOCT1-expressing CCA cells showed higher ability to take up (Fig. 6G-I) and respond (Fig. 6J-L) to sorafenib. Enhanced cytotoxic effect of sorafenib in CCA cells overexpressing hOCT1 was consistent with the inhibition of the phosphorylation of the sorafenib-known target signal transducer and activator of transcription 3 (STAT3) as revealed by immunoblot analysis (Supporting Fig. S4).

\section{TUMOR-SELECTIVE CHEMOSENSITIZATION BY GENE THERAPY}

In order to restrict transgene expression to tumor cells, we designed an adenoviral vector in which hOCT1 expression was driven by BIRC5pr, due to the high activity of this promoter found in clinical CCA specimens. ${ }^{(24)}$ As it has been reported ${ }^{(16)}$ and we have confirmed here, the levels of both BIRC5 mRNA and survivin protein were greatly increased in CCA cells compared with healthy liver and normal human cholangiocytes (Fig. 7A,B). Promoter activity was evaluated in transfection experiments with Alexander cells using vectors encoding firefly luciferase (Luc2) under the control of either $C M V p r$ or BIRC5pr. Both promoters were similarly potent in stimulating Luc2 expression (Fig. 7C,D). Based on these results, adenoviruses bearing BIRC5pr-EGFP (Ad-MOCK) (Fig. 7E) or BIRC5pr-hOCT1-EGFP (Ad-hOCT1) (Fig. 7F) were used to treat nude mice with intrahepatic CCA xenograft (Fig. 8). Gene therapy resulted in selective overexpression of hOCT1 at the plasma membrane of tumor cells, whereas no detectable expression in adjacent peritumor tissue (Fig. 8C-F) or endothelial cells (Supporting Fig. S5) was found. In mice treated with Ad-MOCK, sorafenib treatment failed to affect tumor growth (Fig. 8G,H). In contrast, coadministration of sorafenib plus Ad-hOCT1 resulted in a marked antitumor effect (Fig. 8G,H), which was not accompanied by decreased vascularization (Supporting Fig. S6). Interestingly, in the absence of treatment with sorafenib, the administration of these vectors did not affect tumor growth. Moreover, 

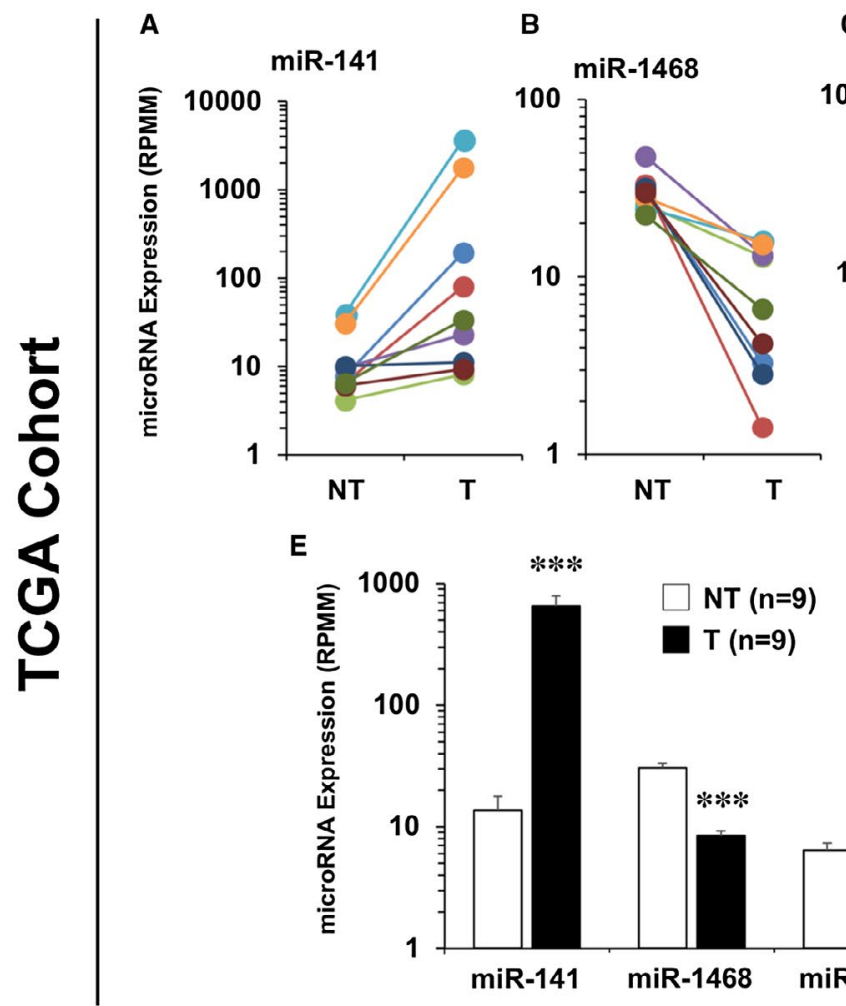

C

$100 \mathrm{miR}^{\mathrm{miR}-30}$

\section{D}
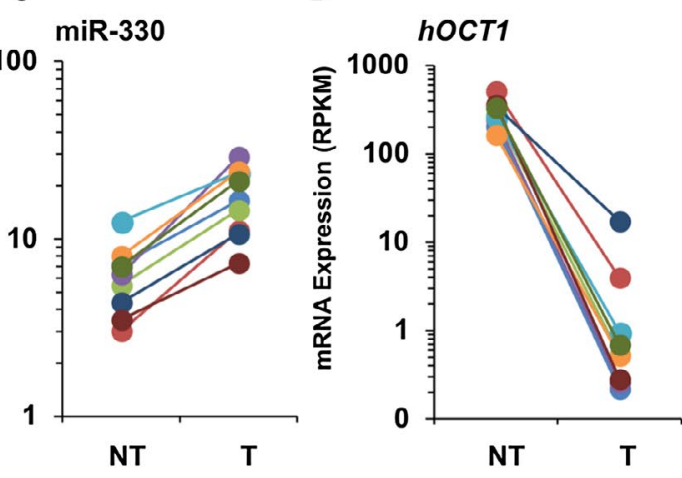

E

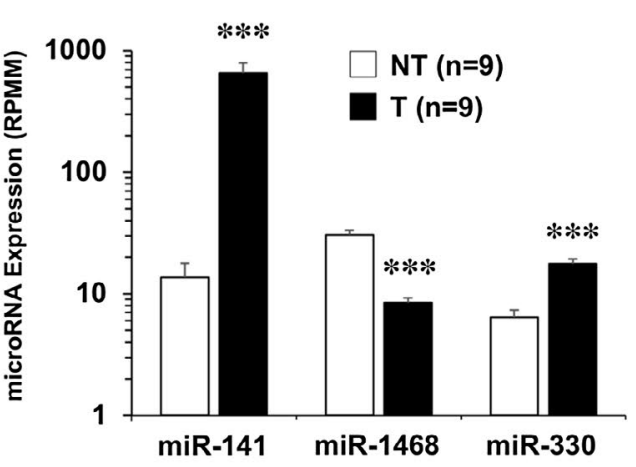

F
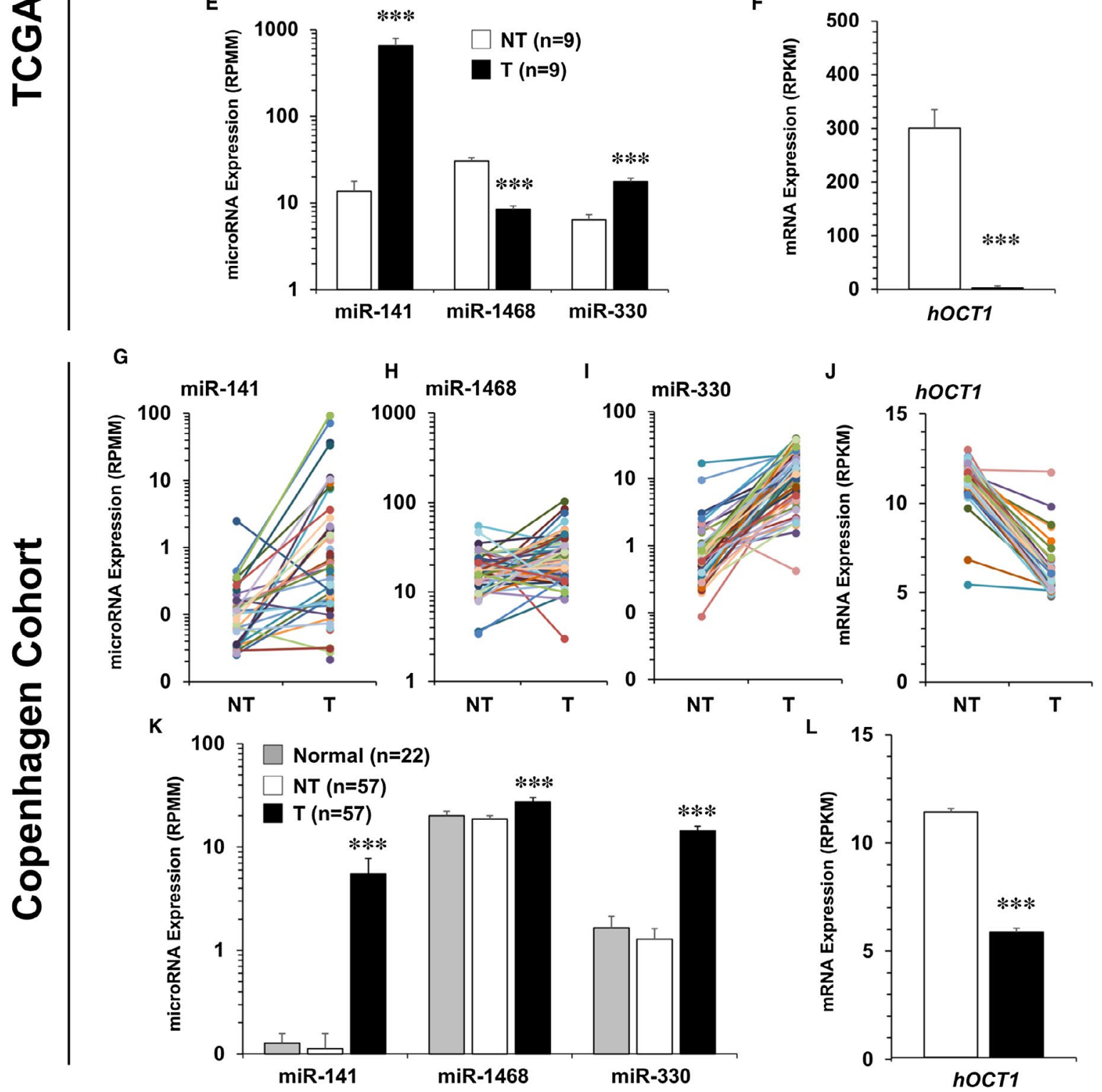
FIG. 3. Expression of microRNAs with potential role in hOCT1 mRNA modulation in CCA. (A-F) Individual values of expression levels of (A) miR-141, (B) miR-1468, (C) miR-330, and (D) bOCT1 mRNA in nine paired samples of tumor (T) tissue and adjacent non-tumor (NT) tissue as downloaded from TCGA-CHOL. (G-L) Similar analysis was carried out on 57 paired T and NT samples and 22 normal livers included in the Copenhagen cohort. Average values (mean \pm SEM) and statistical comparisons are shown in E, F, $\mathrm{K}$, and L. ${ }^{* * *}, P<0.001$, on comparing T and NT by paired $t$ test. Abbreviations: RPKM, reads per kilobase mapped; RPMM, reads per million miRNA mapped.

A
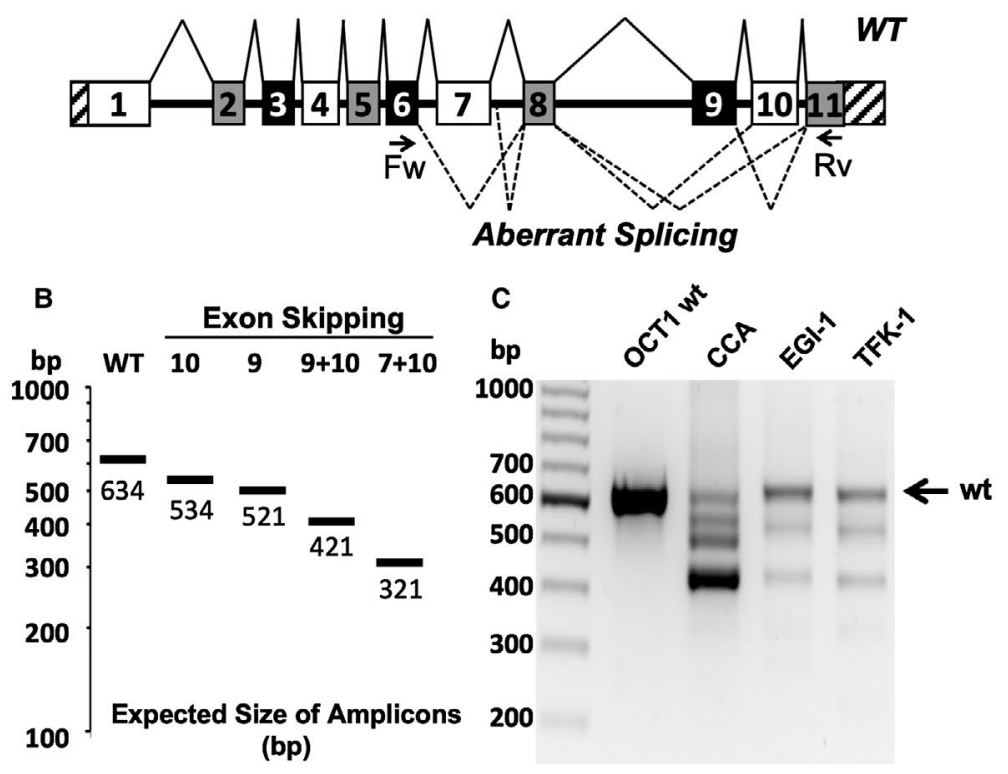

human SLC22A1 Pre-mRNA

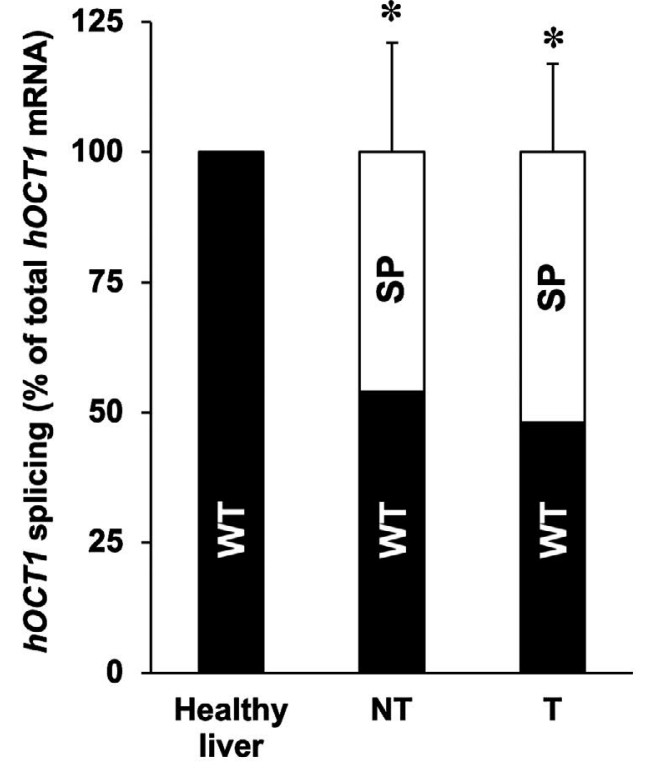

FIG. 4. Alternative splicing of human bOCT1 mRNA in CCA. Scheme of human OCT1 pre-mRNA showing exons (1-11 boxes), introns (horizontal lines), and untranslated regions (striped boxes). Dashed lines indicate exon skipping or intron retention variants due to aberrant splicing. (A) The locations of $\mathrm{Fw}_{\mathrm{w}}$ and $\mathrm{Rv}$ primers used to detect spliced forms are depicted. (B) Expected size of amplicons resulting from PCR using Fw and Rv primers. (C) Representative separation by gel electrophoresis of PCR products obtained using as template: complete $h O C T 1$ mRNA (from a plasmid), CCA tumor tissue, and CCA cells (EGI-1 and TFK-1). (D) Densitometric analysis of the semiquantitative PCR of spliced forms of $h O C T 1 \mathrm{mRNA}$ in healthy liver, paired peritumor tissue (NT, n = 5), and CCA tumor tissue $(\mathrm{T}, \mathrm{n}=9)$. * $P<0.05$, on comparing $\mathrm{T}$ or NT with healthy liver. Comparison of $\mathrm{T}$ with NT was $P>0.05$. Abbreviations: Fw, forward; Rv, reverse; SP, aberrant splicing; WT, wild-type OCT1.

signs of renal or hepatic toxicity were not found (Supporting Table S4).

\section{Discussion}

At present, sorafenib is the reference drug used in the pharmacological treatment of HCC. The high proportion of genetic alterations in critical signaling pathways involved in cell proliferation in CCA has led to an interest in the development of clinical trials investigating targeted therapies including sorafenib. ${ }^{(1)}$ However, data on the effectiveness of sorafenib, and other TKIs, in patients with CCA are controversial, reporting both beneficial effect ${ }^{(12,25,26)}$ and poor activity against this type of liver cancer. ${ }^{(27,28)}$ The latter is not surprising considering the strong multidrug resistance (MDR) phenotype of CCA, in which several MOCs involved in the lack of response to sorafenib have been identified. ${ }^{(5)}$ These include the overexpression of $\mathrm{ABC}$ proteins, such as MDR1 and BCRP, which reduce intracellular drug content (MOC-1b); enhanced drug inactivation by uridine glucuronosyl transferase 1A (MOC-2); or the appearance of genetic variants in the intracellular targets of sorafenib (MOC-3). ${ }^{(29)}$ In addition, we have reported that because cell uptake, which is mediated mainly by hOCT1, is an essential requirement for sorafenib 

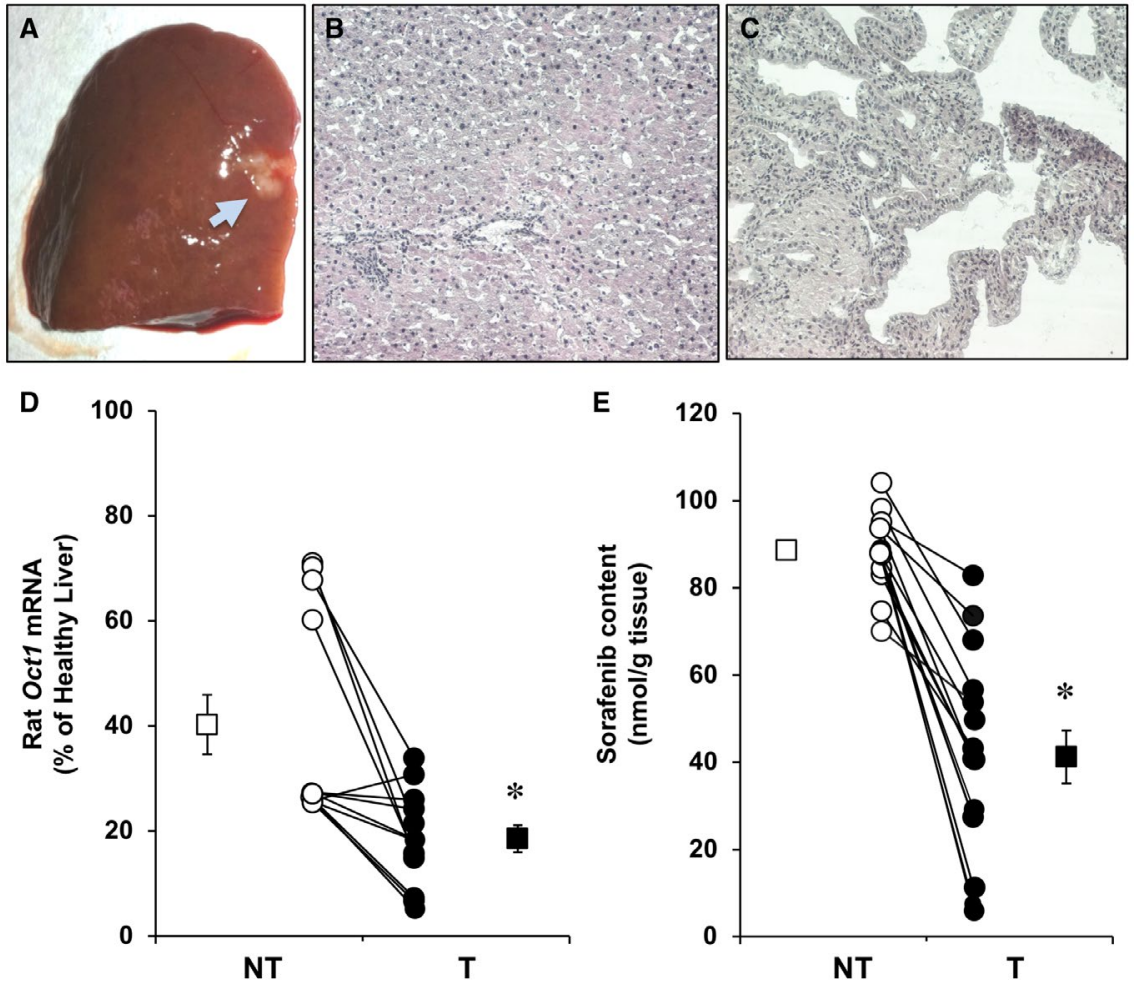

FIG. 5. Relationship between $r O c t 1$ expression and sorafenib uptake in rat CCA. Macroscopic view of (A) tumors (arrow) and histological images by hematoxylin and eosin staining under light-field microscope with $\times 10$ magnification of (B) adjacent liver parenchyma and $(\mathrm{C})$ tumor tissue in rats after 30 weeks of continuous administration of $0.05 \%$ TAA in the drinking water. (D) Relative expression of $r O c t 1$ in tumor $(\mathrm{T})$ and paired non-tumor $(\mathrm{NT})$ liver tissue of rats $(\mathrm{n}=12)$ with chemically induced CCA. (E) Sorafenib content (measured by HPLC-MS/MS) in T $(\mathrm{n}=15)$ and NT $(\mathrm{n}=10)$ tissues 60 minutes after administration of sorafenib $(10 \mathrm{mg} / \mathrm{kg}$ body weight, intravenously). Results are shown as individual values (circles) or as means $\pm \mathrm{SD}$ (squares). * $P<0.05$, on comparing $\mathrm{T}$ with NT.

to reach its intracellular targets and carry out its pharmacological effect, changes in the expression/ activity of this carrier can lead to poorer response to sorafenib. ${ }^{(15)}$ As a natural continuation of this line of research, in the present study, we have analyzed the causes of hOCT1 down-regulation in CCA, and we have explored strategies to restore its expression and hence increase its sensitivity to sorafenib.

We have reported that hOCT1 expression is reduced in $\mathrm{HCC}^{(16)}$ and CCA. ${ }^{(30)}$ In CCA, epigenetic abnormalities, including DNA hypermethylation, have been described. ${ }^{(31)}$ Moreover, hypermethylation of SLC22A1 promoter in HCC has been reported ${ }^{(32)}$ and, as we have demonstrated here, these changes also occur in CCA. An inverse behavior regarding bOCT1 mRNA expression and hypermethylation status of three critical $\mathrm{CpG}$ regions (5'-UTR, transcriptional start site, and exon 1) has been found. This is consistent with the concept that methylation of the first exon correlates with transcriptional silencing of SLC22A1 gene. ${ }^{(33)}$ Although further studies are needed, our data suggest that methylation of $S L C 22 A 1$ could be a prognostic biomarker in CCA, as has been proposed in HCC. ${ }^{(32)}$

As suggested by our results, DAC treatment is an efficient strategy to restore OCT1 expression in CCA cell lines, which enhances sorafenib uptake. These findings support that demethylating agents could be useful in restoring hOCT1 expression in CCA tumors and hence improving sorafenib uptake/response. Moreover, clinical data indicate that DAC treatment retards CCA tumor growth. ${ }^{(34)}$ Thus, combination therapy of DAC plus sorafenib should be explored in patients with CCA. In support of this view, treatment of acute myeloid leukemia with DAC plus sorafenib has shown synergistic antitumor effect. ${ }^{(35)}$ 


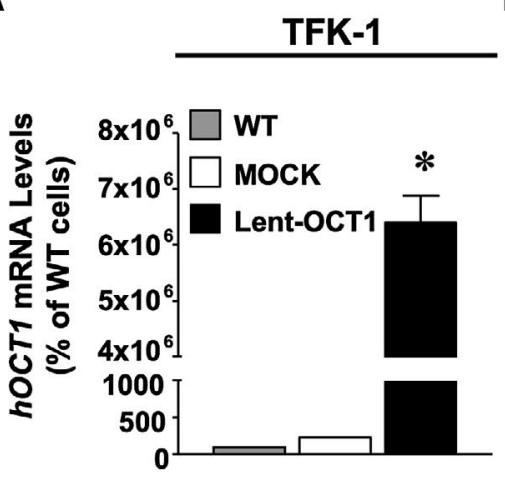

D

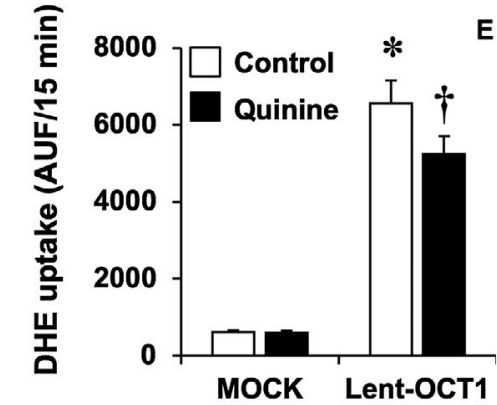

G

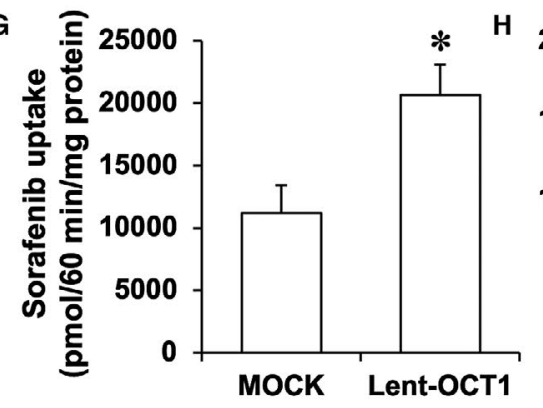

$\mathbf{J}$

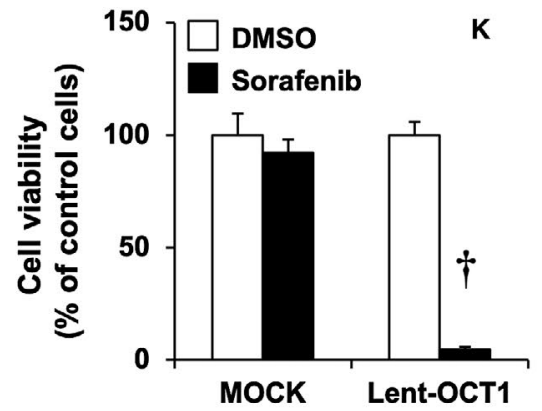

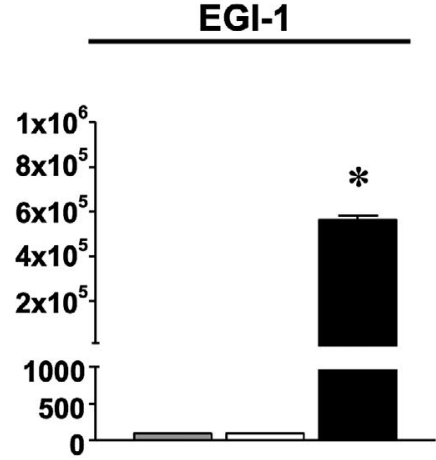

EGI-1

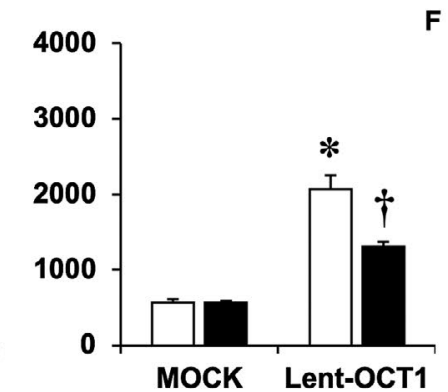
HuCCT1
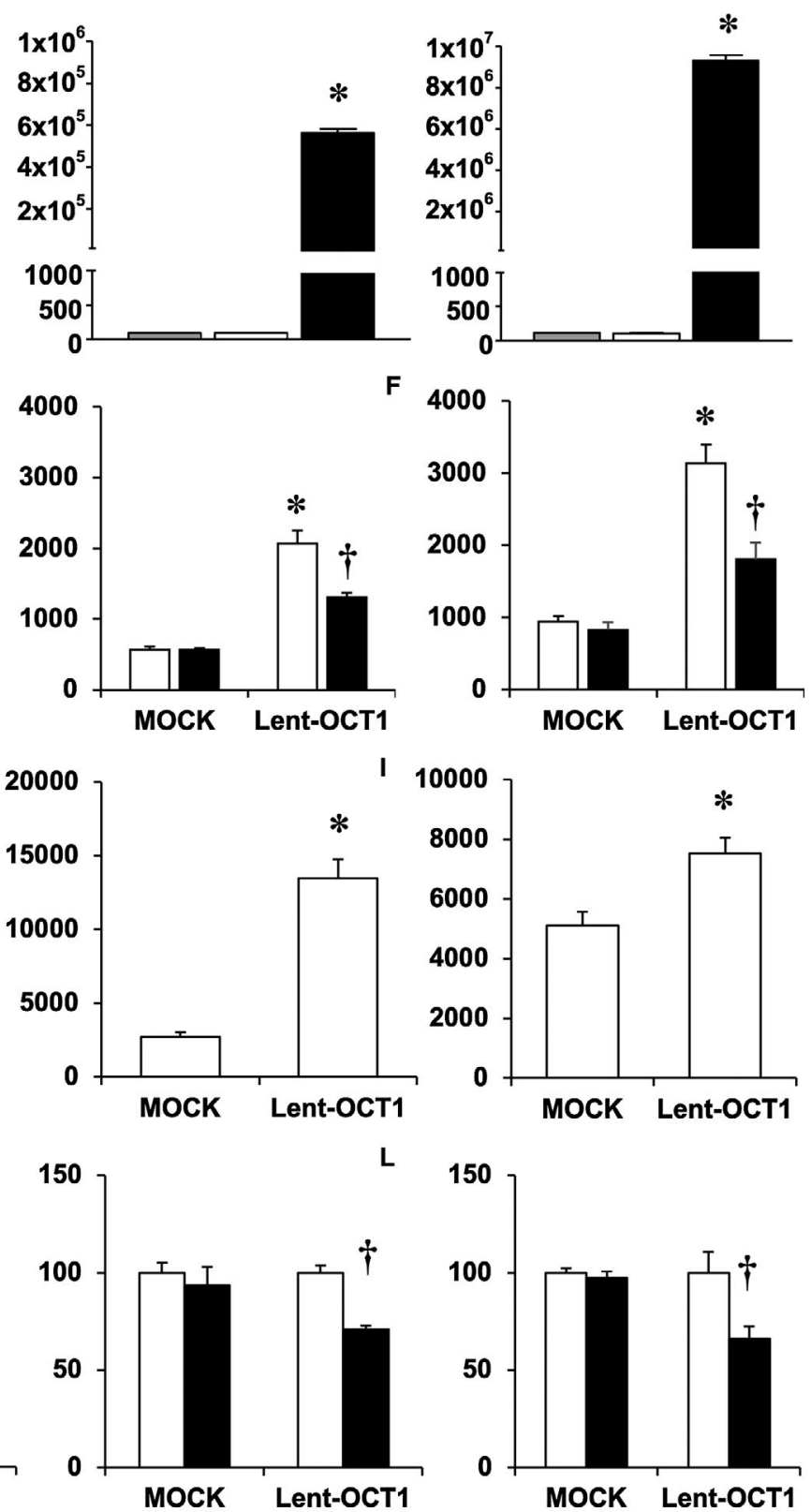

FIG. 6. Role of hOCT1 in sorafenib uptake and response in vitro. Extrahepatic (TFK-1 and EGI-1) and intrahepatic (HuCCT1) CCA cells were transduced with control lentivirus (MOCK) or hOCT1 lentivirus (Lent-OCT1). (A-C) Relative bOCT1 mRNA levels were evaluated in WT-, MOCK-, or OCT1-transduced cells. Measurements were carried out 4 days after transduction. (D-F) MOCK or Lent-OCT1 CCA cells were incubated with $5 \mu \mathrm{M}$ DHE in the absence (Control) or the presence of $250 \mu \mathrm{M}$ quinine for 15 minutes to determine hOCT1 function. (G-I) MOCK or Lent-OCT1 CCA cells were incubated with $5 \mu \mathrm{M}$ sorafenib for 1 hour, and its levels in the cells were measured by HPLC-MS/MS. (J-L) Cell viability was evaluated in MOCK or Lent-OCT1 CCA after incubation with 5 $\mu \mathrm{M}$ sorafenib for 6 hours, and cell viability was measured 66 hours later to evaluate sorafenib response. Values are mean \pm SD from nine wells of three different cultures in each cell line. *, $P<0.05$, on comparing with MOCK cells. $\dagger, P<0.05$, on comparing cells incubated with (D-F) quinine or (J-L) sorafenib with their corresponding Control/DMSO groups. AUF, arbitraty units of fluorescence. 

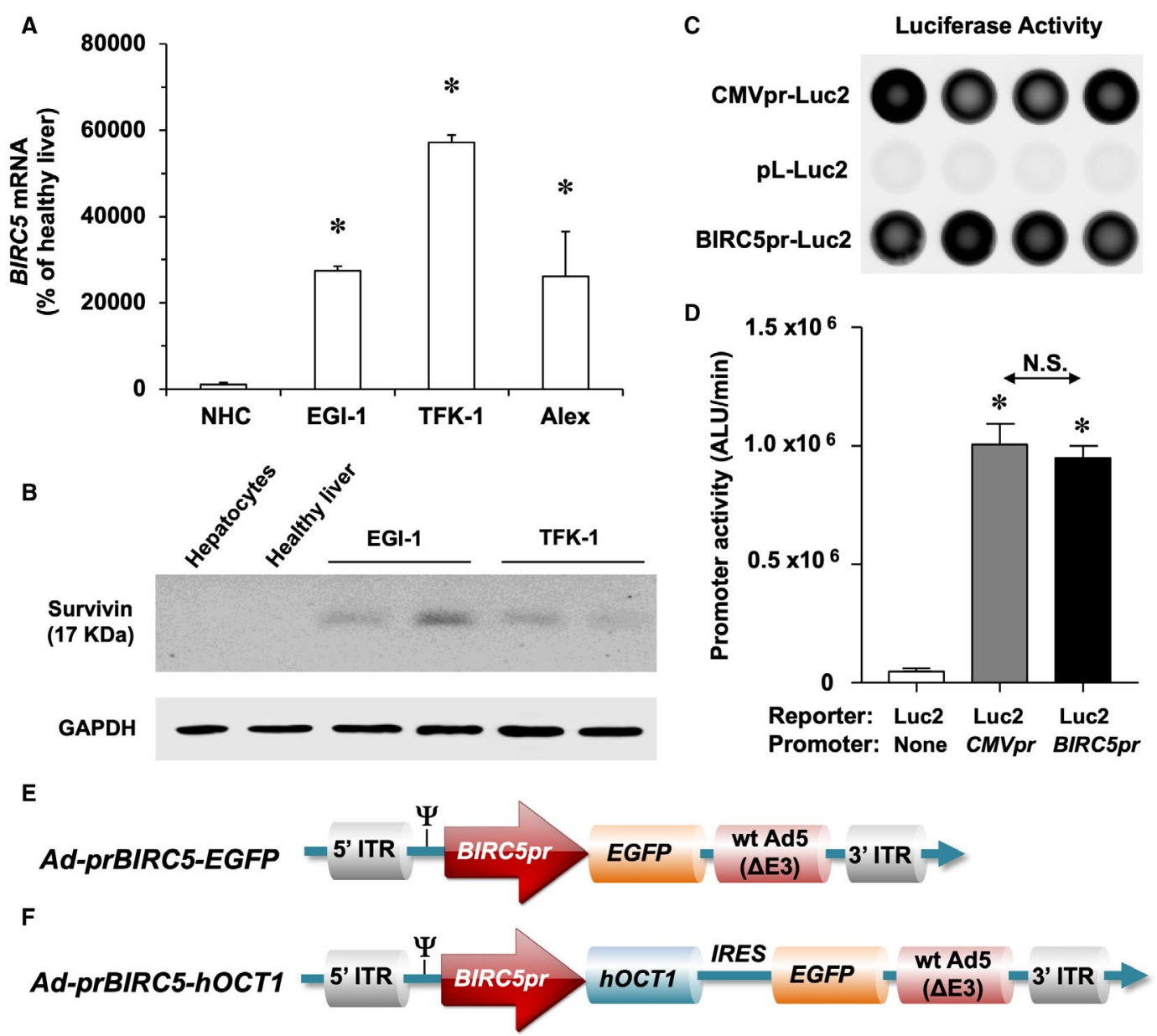

FIG. 7. Tumor-selective gene therapy strategy design. (A) Relative levels of BIRC5 mRNA in NHC, EGI-1, and TFK-1 CCA cells, and in Alexander HCC cells (Alex). (B) Representative immunoblots of survivin and GAPDH in human hepatocytes, healthy liver, and CCA cell lines (EGI-1 and TFK-1). Representative (C) experiment and (D) average values of BIRC5 promoter (BIRC5pr) activity determined 48 hours after transient transfection of Alexander cells with BIRC5pr-Luc2, CMVpr-Luc2, or $p L-L u c 2$ (negative control, without promoter) plasmids. Values are mean $\pm \mathrm{SD}$ from at least three experiments performed in triplicate. * $P<0.01$, as compared with pL-Luc2. Partial scheme of (E) control adenovirus Ad-prBIRC5-EGFP (Ad-MOCK) and (F) adenoviral vector containing hOCT1 ORF (Ad-prBIRC5-hOCT1). Abbreviations: ALU, arbitrary luminescence units; NHC, normal human cholangiocytes; N.S., no significant differences.

Although the up-regulation induced by DAC in EGI-1 cells was strong $(\approx 15$-fold) (Fig. $2 \mathrm{~A})$, the impact of this change in the functional experiments was modest (Fig. 2E). This could be partly due to the fact that a fraction of the synthesized hOCT1 pre-mRNA was processed to inactive variants by an aberrant splicing that was quantitatively important in both CCA tumors and cell lines (Fig. 4C). In a previous study on a low number of specimens of CCA, several variants of aberrant splicing of bOCT1 premRNA were identified. ${ }^{(15)}$ This is pharmacologically relevant because these variants encode truncated nonfunctional proteins. Interestingly, here we have found aberrant variants in both tumor and peritumor tissues, whereas this was negligible in healthy liver. The presence of enhanced alternative splicing in the mRNA of some genes has been described to occur during early stages of several liver diseases. ${ }^{(36)}$ An ongoing study on liver samples from patients with different liver diseases, without cancer, suggests a heterogeneous degree of aberrant splicing of hOCT1 pre-mRNA, which is lower than in CCA (data not shown). 

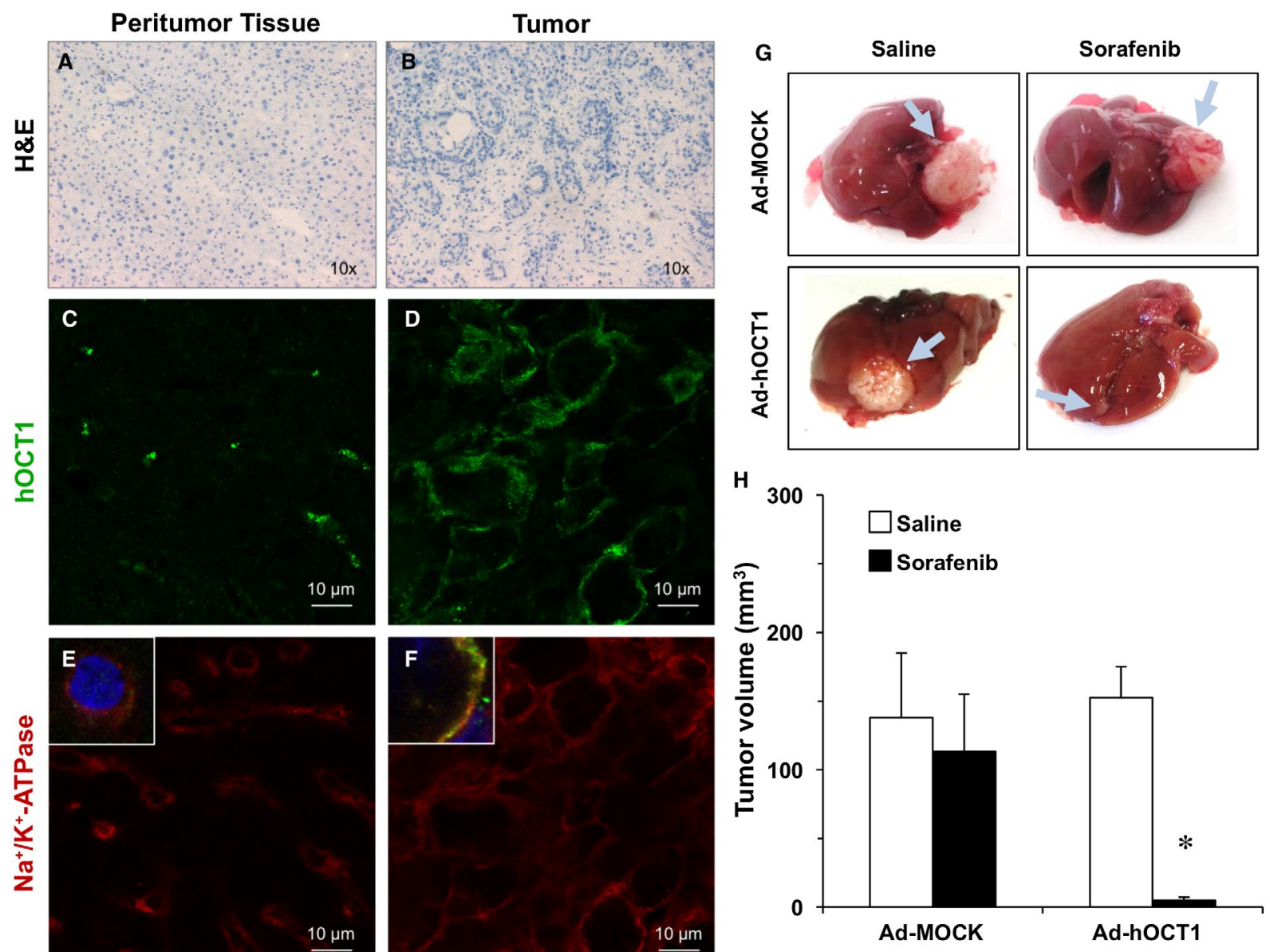

FIG. 8. Antitumor effect of sorafenib on xenograft CCA tumors selectively expressing hOCT1. Nude mice were used to generate subcutaneous CCA tumors with EGI-1 cells. These were used as donors for subsequent intrahepatic implantation in different animals. Histological appearance of adjacent (A) nontumor tissue and (B) CCA tumor tissue as observed by hematoxylin and eosin staining under light-field microscope with $\times 10$ magnification. These animals were treated with adenoviruses injected every 5 days (intravenously through tail vein at $4 \times 10^{7} \mathrm{VP} /$ mice in each administration). (C,D) Adenoviral vector $A d-p r B I R C 5-b O C T 1$ was used to induce hOCT1 expression as seen by immunofluorescence detection by confocal microscopy (cyanin-5 fluorescence was artificially converted into green). $\mathrm{Na}^{+} / \mathrm{K}^{+}$-ATPase (red) was used as a plasma membrane marker in adjacent (E) nontumor liver tissue and (F) implanted CCA tumors. The insets are amplified details of merge images showing the nuclei stained with DAPI (blue). Animals injected with Ad-prBIRC5-hOCT1 (Ad-hOCT1) or control Ad-prBIRC5-EGFP (Ad-MOCK) vectors were treated with saline or sorafenib (10 mg/kg body weight, intraperitoneally twice a week). $(\mathrm{G})$ Representative images of gross appearance of livers of a mouse from each group showing the implanted tumor (arrows). (H) Tumor volume was determined at the end of the experimental period ( 2 months), when the animals were slaughtered and tumors were excised and measured. Values are mean \pm SD from four experimental groups; Ad-MOCK + saline ( $\mathrm{n}=5)$, Ad-MOCK + sorafenib ( $\mathrm{n}=6)$, Ad-hOCT1 + saline ( $\mathrm{n}=4)$, Ad-hOCT1 + sorafenib $(\mathrm{n}=7) .{ }^{*}, P<0.05$, on comparing the Ad-hOCT1 group treated with sorafenib with the group that received saline alone. Abbreviation: H\&E, hematoxylin and eosin.

Other epigenetic mechanisms, such as histone deacetylation, seem to also play a role in the modulation of hOCT1 expression, although to a lesser extent. RBPs, which bind AU-rich elements in the 3' UTR of many mRNAs and target them for stabilization or rapid decay, ${ }^{(37)}$ might also be involved in bOCT1 mRNA decay. As the only change observed was a decreased expression of TTP, which participates in mRNA decay, our results do not support a role of RBPs in bOCT1 mRNA down-regulation. However, this cannot be ruled out because the complex regulation of the function of RBPs has not been explored in depth here. In contrast, in vitro activity measurements together with analysis of microRNA expression 
in CCA samples suggest a possible role of miR-141 and miR-330 in hOCT1 mRNA modulation in CCA.

Although epigenetic therapies such as DAC administration may be useful for recovering $h O C T 1$ mRNA levels, the generation of aberrant isoforms and the presence of inactivating SNPs that appear in $\mathrm{CCA}^{(15)}$ would limit the gaining of hOCT1 function. In contrast, in vitro studies clearly demonstrate that enhanced expression of exogenous hOCT1 results in higher sorafenib uptake and sensitivity. One of the mechanisms accounting for sorafenib antitumor activity involves inhibition of survival Janus kinase/STAT3 signaling pathway that includes reduced STAT3 phosphorylation. ${ }^{(10,38)}$ Immunoblot analysis revealed that treatment of TFK-1 cells with sorafenib induced a marked reduction in the proportion of phosphorylated STAT3 only if the cells overexpressed hOCT1 (Supporting Fig. S4).

Prompted by these findings, we have evaluated the alternative of inducing hOCT1 expression by viral transduction in CCA cells. Thus, as a proof of concept, we have assayed an in vivo strategy of gene therapy using adenoviruses, which can infect a broad range of human cells with high gene transfer efficiency ${ }^{(39,40)}$ and have been proposed for the treatment of neoplastic diseases, including CCA. ${ }^{(41)}$ We have used adenoviruses serotype 5 (Ad5), which has become the most popular system in virotherapy. The fact that Ad5 has hepatotropic properties is an advantage for targeting this organ, but it could be a partial obstacle for its successful application in liver tumors such as CCA. To overcome this problem, we have used a transcriptional targeting strategy that exploits the activity of tumor-specific promoters that are preferentially active in tumor cells in comparison with normal cells. ${ }^{(42)}$ Survivin (BIRC5 gene), a member of the inhibitor of apoptosis family that is involved in controlling mitotic progression and preventing cell death, is overexpressed in many cancers, including CCA, but not in normal adult tissues. ${ }^{(43-45)}$ Previous studies have suggested the interest of BIRC5 pr for tumor-targeted therapy ${ }^{(46)}$ because of its high tumor activity, as high as $C M V \operatorname{Pr}$ (Fig. 7), and low activity in healthy cells. ${ }^{(4)}$ This accounts for the specific transduction reached in the experimental model of orthotopic CCA xenograft. Accordingly, the expression of hOCT1 at the plasma membrane of tumor cells dramatically improved the antitumor effect of sorafenib.
Taken together, our study demonstrates that events such as promoter hypermethylation, microRNA-mediated degradation, and aberrant splicing lead to decreased bOCT1 mRNA and sorafenib uptake/response in CCA. Gene therapy able to selectively induce hOCT1 expression in tumor cells, but not in adjacent healthy liver tissue, is a useful chemosensitization strategy to improve the response of CCA to sorafenib.

\section{REFERENCES}

1) Ahn DH, Bekaii-Saab T. Biliary cancer: intrahepatic cholangiocarcinoma vs. extrahepatic cholangiocarcinoma vs. gallbladder cancers: classification and therapeutic implications. J Gastrointest Oncol 2017;8:293-301.

2) Lazaridis KN, Gores GJ. Cholangiocarcinoma. Gastroenterology 2005;128:1655-1667.

3) Banales JM, Cardinale V, Carpino G, Marzioni M, Andersen JB, Invernizzi P, et al. Expert consensus document: Cholangiocarcinoma: current knowledge and future perspectives consensus statement from the European Network for the Study of Cholangiocarcinoma (ENS-CCA). Nat Rev Gastroenterol Hepatol 2016;13:261-280.

4) Khan SA, Davidson BR, Goldin RD, Heaton N, Karani J, Pereira SP, et al. Guidelines for the diagnosis and treatment of cholangiocarcinoma: an update. Gut 2012;61:1657-1669.

5) Marin JJG, Lozano E, Herraez E, Asensio M, Di Giacomo S, Romero MR, et al. Chemoresistance and chemosensitization in cholangiocarcinoma. Biochim Biophys Acta Mol Basis Dis 2018;1864:1444-1453.

6) Valle J, Wasan H, Palmer DH, Cunningham D, Anthoney A, Maraveyas A, et al. Cisplatin plus gemcitabine versus gemcitabine for biliary tract cancer. N Engl J Med 2010;362:1273-1281.

7) Wilhelm S, Carter C, Lynch M, Lowinger T, Dumas J, Smith RA, et al. Discovery and development of sorafenib: a multikinase inhibitor for treating cancer. Nat Rev Drug Discov 2006;5:835-844.

8) Carlomagno F, Anaganti S, Guida T, Salvatore G, Troncone G, Wilhelm SM, et al. BAY 43-9006 inhibition of oncogenic RET mutants. J Natl Cancer Inst 2006;98:326-334.

9) Llovet JM, Ricci S, Mazzaferro V, Hilgard P, Gane E, Blanc JF, et al. Sorafenib in advanced hepatocellular carcinoma. N Engl J Med 2008;359:378-390.

10) Sugiyama $H$, Onuki K, Ishige $K$, Baba N, Ueda T, Matsuda $S$, et al. Potent in vitro and in vivo antitumor activity of sorafenib against human intrahepatic cholangiocarcinoma cells. J Gastroenterol 2011;46:779-789.

11) Huether A, Hopfner M, Baradari V, Schuppan D, Scherubl H. Sorafenib alone or as combination therapy for growth control of cholangiocarcinoma. Biochem Pharmacol 2007;73:1308-1317.

12) LaRocca RV, Hicks MD, Mull L, Foreman B. Effective palliation of advanced cholangiocarcinoma with sorafenib: a twopatient case report. J Gastrointest Cancer 2007;38:154-156.

13) Pan TT, Wang W, Jia WD, Xu GL. A single-center experience of sorafenib monotherapy in patients with advanced intrahepatic cholangiocarcinoma. Oncol Lett 2017;13:2957-2964.

14) Luo $X$, Jia W, Huang $Z$, Li $X$, Xing B, Jiang $X$, et al. Effectiveness and safety of sorafenib in the treatment of unresectable and advanced intrahepatic cholangiocarcinoma: a pilot study. Oncotarget 2017;8:17246-17257. 
15) Herraez E, Lozano E, Macias RI, Vaquero J, Bujanda L, Banales JM, et al. Expression of SLC22A1 variants may affect the response of hepatocellular carcinoma and cholangiocarcinoma to sorafenib. Hepatology 2013;58:1065-1073.

16) Martinez-Becerra P, Vaquero J, Romero MR, Lozano E, Anadon C, Macias RI, et al. No correlation between the expression of FXR and genes involved in multidrug resistance phenotype of primary liver tumors. Mol Pharm 2012;9:1693-1704.

17) Andersen JB, Spee B, Blechacz BR, Avital I, Komuta M, Barbour A, et al. Genomic and genetic characterization of cholangiocarcinoma identifies therapeutic targets for tyrosine kinase inhibitors. Gastroenterology 2012;142:1021-1031 e1015.

18) Farshidfar F, Zheng S, Gingras MC, Newton Y, Shih J, Robertson AG, et al. Integrative genomic analysis of cholangiocarcinoma identifies distinct IDH-mutant molecular profiles. Cell Rep 2017;18:2780-2794.

19) Lozano E, Sanchez-Vicente L, Monte MJ, Herraez E, Briz O, Banales JM, et al. Cocarcinogenic effects of intrahepatic bile acid accumulation in cholangiocarcinoma development. Mol Cancer Res 2014;12:91-100.

20) Dominguez MF, Macias RI, Izco-Basurko I, de La Fuente A, Pascual MJ, Criado JM, et al. Low in vivo toxicity of a novel cisplatin-ursodeoxycholic derivative (Bamet-UD2) with enhanced cytostatic activity versus liver tumors. J Pharmacol Exp Ther 2001;297:1106-1112.

21) Markwell MA, Haas SM, Bieber LL, Tolbert NE. A modification of the Lowry procedure to simplify protein determination in membrane and lipoprotein samples. Anal Biochem 1978;87:206-210.

22) Morine $Y$, Shimada $M$, Iwahashi $S$, Utsunomiya $T$, Imura $S$, Ikemoto $\mathrm{T}$, et al. Role of histone deacetylase expression in intrahepatic cholangiocarcinoma. Surgery 2012;151:412-419.

23) Al-Abdulla R, Lozano E, Macias RIR, Monte MJ, Briz O, O'Rourke CJ, et al. Epigenetic events involved in organic cation transporter 1-dependent impaired response of hepatocellular carcinoma to sorafenib. Br J Pharmacol 2019;176:787-800.

24) Zhu ZB, Chen Y, Makhija SK, Lu B, Wang M, Rivera AA, et al. Survivin promoter-based conditionally replicative adenoviruses target cholangiocarcinoma. Int J Oncol 2006;29:1319-1329.

25) Pinter M, Sieghart W, Reisegger M, Wrba F, Peck-Radosavljevic M. Sorafenib in unresectable intrahepatic cholangiocellular carcinoma: a case report. Wien Klin Wochenschr 2011;123:61-64.

26) Chakunta HR, Sunderkrishnan R, Kaplan MA, Mostofi R. Cholangiocarcinoma: treatment with sorafenib extended life expectancy to greater than four years. J Gastrointest Oncol 2013;4:E30-E32.

27) El-Khoueiry AB, Rankin CJ, Ben-Josef E, Lenz HJ, Gold PJ, Hamilton RD, et al. SWOG 0514: a phase II study of sorafenib in patients with unresectable or metastatic gallbladder carcinoma and cholangiocarcinoma. Invest New Drugs 2012;30:1646-1651.

28) Bengala C, Bertolini F, Malavasi N, Boni C, Aitini E, Dealis C, et al. Sorafenib in patients with advanced biliary tract carcinoma: a phase II trial. Br J Cancer 2010;102:68-72.

29) Rosenzweig SA. Acquired resistance to drugs targeting receptor tyrosine kinases. Biochem Pharmacol 2012;83:1041-1048.

30) Lozano E, Monte MJ, Briz O, Hernandez-Hernandez A, Banales JM, Marin JJ, et al. Enhanced antitumour drug delivery to cholangiocarcinoma through the apical sodium-dependent bile acid transporter (ASBT). J Control Release 2015;216:93-102.

31) Jusakul A, Cutcutache I, Yong CH, Lim JQ, Huang MN, Padmanabhan N, et al. Whole-genome and epigenomic landscapes of etiologically distinct subtypes of cholangiocarcinoma. Cancer Discov 2017;7:1116-1135.
32) Schaeffeler E, Hellerbrand C, Nies AT, Winter S, Kruck S, Hofmann U, et al. DNA methylation is associated with downregulation of the organic cation transporter OCT1 (SLC22A1) in human hepatocellular carcinoma. Genome Med 2011;3: 82.

33) Brenet F, Moh M, Funk P, Feierstein E, Viale AJ, Socci ND, et al. DNA methylation of the first exon is tightly linked to transcriptional silencing. PLoS One 2011;6:e14524.

34) Wang B, Li H, Yang R, Zhou S, Zou S. Decitabine inhibits the cell growth of cholangiocarcinoma in cultured cell lines and mouse xenografts. Oncol Lett 2014;8:1919-1924.

35) Muppidi MR, Portwood S, Griffiths EA, Thompson JE, Ford LA, Freyer CW, et al. Decitabine and sorafenib therapy in FLT-3 ITD-mutant acute myeloid leukemia. Clin Lymphoma Myeloma Leuk 2015;15(Suppl. 15):S73-S79.

36) Webster NJG. Alternative RNA splicing in the pathogenesis of liver disease. Front Endocrinol (Lausanne) 2017;8:133.

37) Sanchez-Diaz P, Penalva LO. Post-transcription meets postgenomic: the saga of RNA binding proteins in a new era. RNA Biol 2006;3:101-109.

38) Blechacz BR, Smoot RL, Bronk SF, Werneburg NW, Sirica AE, Gores GJ. Sorafenib inhibits signal transducer and activator of transcription-3 signaling in cholangiocarcinoma cells by activating the phosphatase shatterproof 2 . Hepatology 2009;50:1861-1870.

39) Alemany R, Gomez-Manzano C, Balague C, Yung WK, Curiel DT, Kyritsis AP, et al. Gene therapy for gliomas: molecular targets, adenoviral vectors, and oncolytic adenoviruses. Exp Cell Res 1999;252:1-12.

40) Curiel DT. Strategies to adapt adenoviral vectors for targeted delivery. Ann N Y Acad Sci 1999;886:158-171.

41) Nagi P, Vickers SM, Davydova J, Adachi Y, Takayama K, Barker $\mathrm{S}$, et al. Development of a therapeutic adenoviral vector for cholangiocarcinoma combining tumor-restricted gene expression and infectivity enhancement. J Gastrointest Surg 2003;7: 364-371.

42) Saukkonen K, Hemminki A. Tissue-specific promoters for cancer gene therapy. Expert Opin Biol Ther 2004;4:683-696.

43) Lu B, Makhija SK, Nettelbeck DM, Rivera AA, Wang M, Komarova S, et al. Evaluation of tumor-specific promoter activities in melanoma. Gene Ther 2005;12:330-338.

44) Chen JS, Liu JC, Shen L, Rau KM, Kuo HP, Li YM, et al. Cancer-specific activation of the survivin promoter and its potential use in gene therapy. Cancer Gene Ther 2004;11: 740-747.

45) Ulasov IV, Rivera AA, Sonabend AM, Rivera LB, Wang M, $\mathrm{Zhu} \mathrm{ZB}$, et al. Comparative evaluation of survivin, midkine and CXCR45 promoters for transcriptional targeting of glioma gene therapy. Cancer Biol Ther 2007;6:679-685.

46) Garg H, Salcedo R, Trinchieri G, Blumenthal R. Improved nonviral cancer suicide gene therapy using survivin promoter-driven mutant Bax. Cancer Gene Ther 2010;17:155-163.

47) Zhu ZB, Makhija SK, Lu B, Wang M, Kaliberova L, Liu B, et al. Transcriptional targeting of tumors with a novel tumor-specific survivin promoter. Cancer Gene Ther 2004;11:256-262.

Author names in bold designate shared co-first authorship.

\section{Supporting Information}

Additional Supporting Information may be found at onlinelibrary.wiley.com/doi/10.1002/hep.30656/suppinfo. 